\title{
THREE-DIMENSIONAL SIMULATIONS OF THE PARKER INSTABILITY IN A UNIFORMLY ROTATING DISK
}

\author{
Jongsoo KIM, ${ }^{1}$ DONGSU RyU, ${ }^{2}$ AND T. W. JonES ${ }^{3}$ \\ Received 2000 October 5 ; accepted 2001 April 10
}

\begin{abstract}
We investigate the effects of rotation on the evolution of the Parker instability by carrying out threedimensional numerical simulations with an isothermal magnetohydrodynamic code. These simulations extend our previous work on the nonlinear evolution of the Parker instability by J. Kim and coworkers. The initial equilibrium system is composed of exponentially stratified gas and a field (along the azimuthal direction) in a uniform gravity (along the downward vertical direction). The computational box, placed at the solar neighborhood, is set to rotate uniformly around the Galactic center with a constant angular speed. The instability has been initialized by random velocity perturbations. In the linear stage, the evolution is not much different from that without rotation, and the mixed (undular + interchange) mode regulates the system. The interchange mode induces alternating dense and rarefied regions with small radial wavelengths, while the undular mode bends the magnetic field lines in the plane of the azimuthal and vertical directions. In the nonlinear stage, flow motion overall becomes chaotic, as in the case without rotation. However, as the gas in higher positions slides down along field lines forming supersonic flows, the Coriolis force becomes important. As oppositely directed flows fall into valleys along both sides of the magnetic field lines, they experience the Coriolis force toward opposite directions, which twists the magnetic field lines there. Hence, we suggest that the Coriolis force plays a role in randomizing the magnetic field. The three-dimensional density structure formed by the instability is still sheetlike with the short dimension along the radial direction, as in the case without rotation. However, the long dimension is now slightly tilted with respect to the mean field direction. The shape of highdensity regions is a bit rounder. The maximum enhancement factor of the vertical column density relative to its initial value is about 1.5 , which is smaller than that in the case without rotation. We conclude that uniform rotation does not change our point of view that the Parker instability alone is not a viable mechanism for the formation of giant molecular clouds.
\end{abstract}

Subject headings: instabilities - ISM: clouds - ISM: magnetic fields - ISM: structure - MHD

On-line material: color figures

\section{INTRODUCTION}

The nature of the Galactic magnetic field has been revealed from observations of pulsar dispersion measures, starlight polarization, and synchrotron emission (Heiles 1996 and references therein). It is composed of uniform and random components. Their estimated strengths at the solar neighborhood are on the order of a few microgauss. The scale of the irregularities of the random component measured by Rand \& Kulkarni (1989) is about $\sim 50$ pc. The magnetic energy density is comparable to those of other energy contents such as cosmic rays, turbulence, and gravity. Therefore, the magnetic field forms one of the important constituents of the interstellar medium (ISM) and plays a key role in its evolution. Parker (1966) demonstrated that the ISM is unstable because of magnetic buoyancy. The instability, referred to as the Parker instability, is an example demonstrating that the magnetic field is important in the dynamics of the ISM.

Two different approaches are employed to investigate the nature of instabilities. One is the linear stability analysis,

\footnotetext{
${ }^{1}$ Korea Astronomy Observatory, 61-1, Hwaam-Dong, Yusong-Ku, Taejon 305-348, Korea; and National Center for Supercomputing Applications, University of Illinois at Urbana-Champaign, 405 North Mathews Avenue, Urbana, IL 61801; jskim@kao.re.kr, jskim@ncsa.uiuc.edu.

${ }^{2}$ Department of Astronomy and Space Science, Chungnam National University, Daejeon 305-764, Korea; ryu@canopus.chungnam.ac.kr.

${ }^{3}$ Department of Astronomy, University of Minnesota, 116 Church Street, SE, Minneapolis, MN 55455; twj@mail.msi.umn.edu.
}

which enables us to find unstable modes and estimate their growth rate. Parker $(1966,1967)$ himself used this approach to show the existence of the instability. Later, other authors elaborated on the instability with the same approach. A partial list includes the studies on the effects of Galactic rotation (Shu 1974; Zweibel \& Kulsrud 1975; Foglizzo \& Tagger 1994, 1995), magnetic microturbulence (Zweibel \& Kulsrud 1975), a skewed configuration of the magnetic field (Hanawa, Matsumoto, \& Shibata 1992), a nonuniform nature of externally given gravity (Horiuchi et al. 1988; Giz \& Shu 1993; Kim, Hong, \& Ryu 1997; Kim \& Hong 1998), the Galactic corona (Kamaya et al. 1997), and multiple gas components (Kim et al. 2000).

According to the linear analysis, the instability is broken down into two distinct modes: undular and interchange (Hughes \& Cattaneo 1987; Kim et al. 1998). The former, whose perturbations are defined in the plane of the gravitational field and the unperturbed magnetic field, undulates magnetic field lines and induces gas to slide down along the field lines into magnetic valleys. The latter interchange mode, whose perturbations are defined in the plane perpendicular to the direction of the unperturbed magnetic field, generates alternating dense and rarefied regions along the radial direction perpendicular to both the gravity and field directions and has a maximum growth rate at an infinite radial wavenumber, indicating that it is a Rayleigh-Taylor type. When perturbations are allowed in all three spatial dimensions, the mixed mode of the undular and interchange modes appears (Matsumoto et al. 1993), which has a 
maximum growth rate at an infinite radial wavenumber too.

The other approach is through numerical simulations, in which the nonlinear evolution of instabilities is studied. Since the first one-dimensional study by Baierlein (1983), many studies of the Parker instability under different conditions have been done: Matsumoto et al. (1990) and Matsumoto \& Shibata (1992) explored the nature of the undular and mixed modes in accretion disk environments. Shibata et al. (1989a, 1989b), Kaisig et al. (1990), and Nozawa et al. (1992) considered the Parker instability as the driving mechanism for emergent flux tubes in the Sun and tried to explain several features of the solar activity. Basu, Mouschovias, \& Paleologou (1997) and Kim et al. (1998) performed multidimensional simulations of the instability in a thin gaseous disk under the influence of a simplified uniform gravity and addressed the issue of the origin of the giant molecular clouds (GMCs). Santillán et al. (2000) simulated the evolution of the Parker instability in a more realistic Galactic disk with multiple gas components.

Kim et al. (1998) especially used high-resolution threedimensional simulations to follow the nonlinear evolution of the mixed mode. They found that after linear growth, perturbations become saturated in the nonlinear stage while forming chaotic structures. On top of that, alternating regions of magnetic arches and valleys are developed. The chaotic motions induce reconnection, especially in the valley regions, which allows gas to cross field lines. As a result, the gas and magnetic field are redistributed into a stable system in the relaxed stage.

In this paper we begin to investigate the effects of rotation on the nonlinear Parker instability. For simplicity, we consider uniform rotation. Eventually, it is necessary to address the effects of differential rotation, but we will leave that for a future work.

It is known through linear analyses (Shu 1974; Zweibel \& Kulsrud 1975) that uniform rotation reduces the growth rate of the undular mode. It is the Coriolis force that prevents the lateral motion and reduces the growth rate. However, as shown in $\S 2$, the Coriolis force affects the linear growth of the three-dimensional mixed mode less. The reason is the following: The normal modes have small wavelengths along the radial direction, and the most unstable mode has a vanishing wavelength. Hence, the perturbed velocity in the linear stage is predominantly along the vertical direction, the direction of the rotation vector, so it does not interfere with the rotation, but yet, from this, it is not clear how the Coriolis force affects the nonlinear evolution. Related to that, Chou et al. (1997) studied rotating flux tubes numerically, modeling the evolution of the emergent magnetic flux sheet. They demonstrated that the emergent flux sheet becomes twisted because of the Coriolis force and explained the tilted active regions observed in the Sun in terms of twisted flux sheets. They, however, chose perturbations with a pair of finite horizontal wavelengths that do not correspond to the mode of the maximum growth rate, and they performed numerical experiments with low resolution. Miller \& Stone (2000) studied in the environment of accretion disks the effects of differential rotation. They focused on the role of the magnetorotational instability but also commented on the role of the Parker instability too. Here, we study the nonlinear evolution of the Parker instability in the environment of the Galactic disk, which is initiated by random perturbations through high- resolution, three-dimensional numerical simulations of the Parker instability. High resolution and three dimensions are required to resolve the small-scale structures of the mixed mode. Random perturbations are necessary to find the mode preferred by the system.

The Parker instability has been thought to be a mechanism in forming the GMCs in the Galaxy (Appenzeller 1974; Mouschovias, Shu, \& Woodward 1974; Blitz \& Shu 1980; Shibata \& Matsumoto 1991; Handa et al. 1992; Gomez de Castro \& Pudritz 1992). The conjecture is based on the results from two-dimensional linear analyses of the undular mode that the growth time and wavelength of the most unstable mode are comparable to the lifetime and the separation of the GMCs. However, it has been known from earlier work by Parker (1967) that the instability initiated by three-dimensional perturbations develops the mixed mode at its maximum growth rate with vanishing wavelength along the radial direction. In addition, Kim et al. (1998) showed in three-dimensional simulations that sheetlike structures with the smallest scale along the radial direction, whose shape is different from that of observed GMCs, form at the developing stage of the instability and persist during the nonlinear stage. Furthermore, they showed that the enhancement factor of the column density relative to its initial value is only $\sim 2$, which is too small for the GMC formation. We will revisit the issue of the GMC formation by the Parker instability with the results on the effects of rotation on the instability.

The plan of the paper is as follows: In $\S 2$ we summarize the problem including numerical set up and linear analysis. In $\S 3$ the results of numerical simulations are presented. Summary and discussion follow in $\S 4$.

\section{PROBLEM}

\subsection{Numerical Setup}

In order to describe the effects of rotation on the Parker instability at the solar neighborhood, we introduce a local Cartesian coordinate system, which is rotating with the Galactic angular velocity $\Omega \hat{z}$ at the Sun's position $R_{0}$. In this local frame, the coordinates are defined as $x=(R$ $\left.-R_{0}\right), y=R(\phi-\Omega t)$, and $z$, and their directions are parallel to the radial, azimuthal, and vertical directions of the Galaxy, respectively. Assuming a constant angular speed, the isothermal magnetohydrodynamic (MHD) equations with a downward uniform gravity $(-g \hat{z})$ are

$$
\begin{gathered}
\frac{\partial \rho}{\partial t}+\nabla \cdot(\rho \boldsymbol{v})=0, \\
\frac{\partial}{\partial t}(\rho \boldsymbol{v})+\nabla \cdot\left(\rho \boldsymbol{v} \boldsymbol{v}+\rho a^{2} \boldsymbol{I}+\frac{B^{2}}{8 \pi} \boldsymbol{I}-\frac{\boldsymbol{B} \boldsymbol{B}}{4 \pi}\right) \\
=-2 \rho \Omega \hat{z} \times \boldsymbol{v}-\rho g \hat{z}, \\
\frac{\partial \boldsymbol{B}}{\partial t}+\nabla \cdot(\boldsymbol{v B}-\boldsymbol{B} \boldsymbol{v})=0,
\end{gathered}
$$

where $a$ is an isothermal sound speed and the other notations have their usual meanings. Note that we do not take into account the centrifugal force in equation (2) since the force is canceled out by the radial component of the gravitational force. The above equations are the same as those solved in Kim et al. (1998) except for the Coriolis force term in the momentum equation. 
As in Kim et al. (1998), the initial distributions of gas and the magnetic field have been set up in magnetohydrostatic equilibrium. Dropping the terms containing the time derivative and velocity in equation (2) and assuming (1) a unidirectional horizontal magnetic field $B_{0}(z) / \hat{y}$ and (2) a constant ratio of magnetic to gas pressures $\alpha=$ $B_{0}^{2}(z) /\left[8 \pi \rho_{0}(z) a^{2}\right]$, then the density and magnetic pressure are described by an exponential function,

$$
\frac{\rho_{0}(z)}{\rho_{0}(0)}=\frac{B_{0}^{2}(z)}{B_{0}^{2}(0)}=\exp \left(-\frac{|z|}{H}\right)
$$

where the scale height of the gas is defined as $H \equiv(1+\alpha) a^{2} / g$.

A computational cube with $0 \leq x, y, z \leq 12 \mathrm{H}$ is placed at the solar neighborhood, where $12 \mathrm{H}$ is the azimuthal wavelength of the maximum linear growth for the undular mode (see the next subsection). To initiate the instability, random velocity perturbations are added. The standard deviation of each velocity component is set to be $10^{-4} a$. Boundaries are periodic along the radial and azimuthal ( $x$ and $y$ ) directions and reflecting along the vertical $(z)$ direction. As the result, fluid quantities are conserved, and expulsion of the magnetic flux through the upper boundary is prohibited.

The above isothermal MHD equations are solved by an MHD code described in Kim et al. (1999), which is the isothermal version of the MHD code based on the explicit, finite-difference total variation diminishing scheme (Ryu \& Jones 1995; Ryu, Jones, \& Frank 1995). Three-dimensional simulations have been performed for the cases with and without rotation using different resolutions. Model parameters of the simulations are summarized in Table 1. Models 1-3 are the cases without rotation. The highest resolution case, model 3, is the same one as that reported in Kim et al. (1998) except that model 3 extends to a longer end time $t_{\text {end }}$. We note that model 3 does not simply extend that of Kim et al. (1998) but has been recalculated with a different set of initial random numbers. The case with rotation, model 4 , has the angular velocity $\Omega=\frac{1}{2}(a / H)$, which is consistent with the Galactic circular speed $\left(27 \mathrm{~km} \mathrm{~s}^{-1} \mathrm{kpc}^{-1}\right)$ based on Hipparcos data (Feast \& Whitelook 1997). All models are assumed to have $\alpha=1$, so that magnetic pressure is initially the same as gas pressure.

Physical quantities of length, speed, density, and the magnetic field are given in units of the density scale height $H$, the isothermal sound speed $a$, the initial density at $z=0$, $\rho_{0}(0)$, and the initial field strength at $z=0, B_{0}(0)$, respectively. For the typical values of $a=6.4 \mathrm{~km} \mathrm{~s}^{-1}$ and $H=160$ pc (Falgarone \& Lequeux 1973), the resulting time unit $H / a$ becomes $2.5 \times 10^{7} \mathrm{yr}$.

It is well known that the real interstellar medium is composed of multiple components with various scale heights.

TABLE 1

Model Parameters of the Simulations

\begin{tabular}{lccrl}
\hline \hline Model $^{\mathrm{a}}$ & $N_{x} \times N_{y} \times N_{z}$ & Computational Domain $^{\mathrm{b}}$ & $t_{\text {end }}^{\mathrm{c}}$ & $\Omega^{\mathrm{c}}$ \\
\hline $1 \ldots \ldots$. & $64 \times 64 \times 64$ & $0 \leq x, y, z \leq 12$ & 100 & 0 \\
$2 \ldots \ldots$ & $128 \times 128 \times 128$ & $0 \leq x, y, z \leq 12$ & 100 & 0 \\
$3 \ldots \ldots$ & $256 \times 256 \times 256$ & $0 \leq x, y, z \leq 12$ & 93 & 0 \\
$4 \ldots \ldots$ & $256 \times 256 \times 256$ & $0 \leq x, y, z \leq 12$ & 70 & 0.5 \\
\hline
\end{tabular}

a All models have $\alpha=1$ initially.

b The length unit is the scale height $H$.

${ }^{c}$ The time unit is the sound travel time over the scale height $H / a$.
Some of them have scale heights different from our choice, 160 pc (see eq. [5] in Kim et al. 2000). So our single gas component model under uniform gravity would be too simplistic. However, it is the model that has been the most extensively used since the pioneering work done by Parker (1966). The effect of uniform rotation on the instability especially was already investigated through linear analyses using the same model (see next subsection). Choosing the well-studied model would enable us to single out the consequences of rotation on the Parker instability in the numerical simulations, which is the primary goal of this paper. We note that two-dimensional numerical simulations of the Parker instability under a more realistic Galactic disk model were performed in Santillán et al. (2000), as noted in $\S 1$.

\subsection{Stability Analyses}

Criteria for the Parker instability are given in terms of an effective adiabatic index. If the adiabatic index is less than a critical value, the above system becomes unstable (Parker 1966, 1967; Hughes \& Cattaneo 1987; Matsumoto et al. 1993). The critical adiabatic index for the undular mode is $\gamma_{u}=(1+\alpha)^{2} /[1+(3 / 2) \alpha]$, while that for the interchange mode is $\gamma_{i}=1-\alpha$. The critical adiabatic index for the mixed mode is $\gamma_{m}=1+\alpha$. Our consideration of isothermality corresponds to $\gamma=1$, and the models have $\alpha=1$. So the initial system is unstable against the undular mode as well as the mixed mode but stable to the interchange mode.

Dispersion relations from linear stability analyses provide the growth rate and wavelength of unstable modes and help set the size of the computational cube. In Figure 1 the dispersion relations for the exponentially stratified magnetized disk are reproduced (see Parker 1966, 1967; Shu 1974; Zweibel \& Kulsrud 1975). The solid line represents

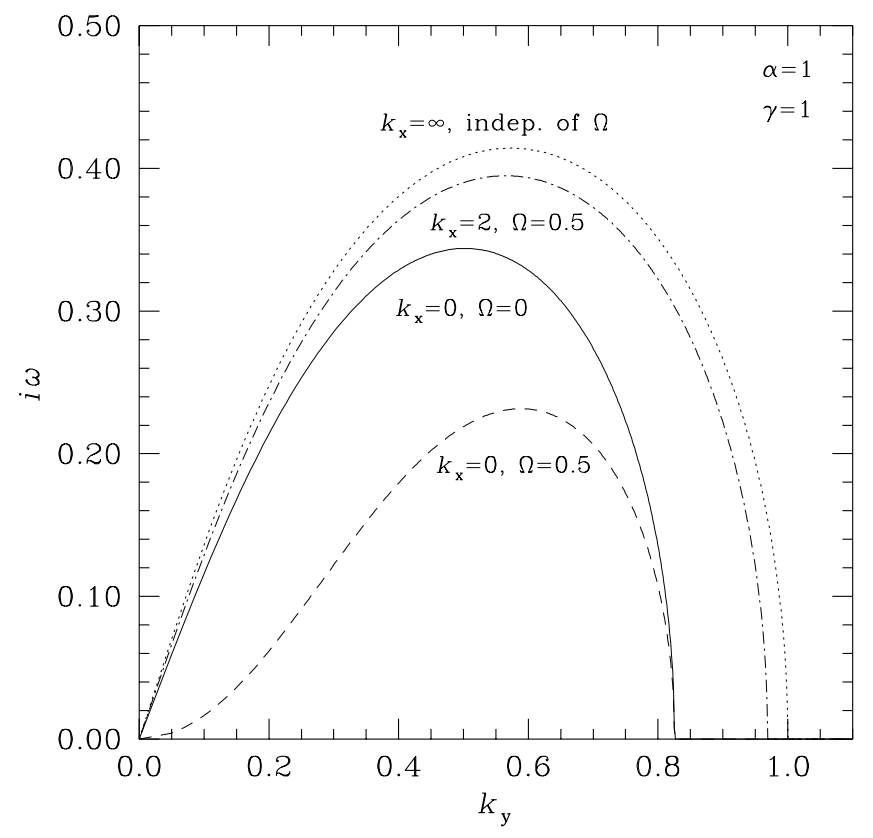

FIG. 1.-Dispersion relations for the Parker instability in exponentially stratified magnetized disks with and without rotation. The ordinate represents the growth rate, and the abscissa represents the azimuthal wavenumber. Each curve is marked by the radial wavenumber and angular speed. The normalized units for the wavenumbers, the growth rate, and the angular speed are $1 / H, a / H$, and $a / H$, respectively, where $H$ is the scale height and $a$ is the isothermal speed. 
the unstable undular mode, which is initiated by twodimensional ( $y$ and $z$ ) perturbations without rotation. Perturbations undulate azimuthal field lines and induce gas to slide down along the field lines into magnetic valleys. The undular mode has a preferred wavelength $(\sim 12 \mathrm{H})$ along the azimuthal direction, which has been set as the size of our computational cube. Inclusion of rigid-body rotation in the undular mode reduces the growth rate as well as the wavelength of the most unstable mode (dashed line) because lateral motion along the field lines is hampered by the Coriolis force. When additional radial perturbations are applied, both the undular and interchange modes work together, and the resulting mixed (undular + interchange) mode becomes more unstable than the undular mode alone. In the extreme case with an infinite radial wavenumber, the growth rates do not depend on angular speed (dotted line). This is because the perturbations with large radial wavenumbers generate mostly vertical motions, which do not interfere with the Coriolis force. With an infinite radial wavenumber, the growth rate of the most unstable mode is $\sim 0.41$, and its wavelength is $\sim 11 \mathrm{H}$. The dot-dashed line shows the dispersion relation with the radial wavelength equal to $\pi$, about one-quarter of the box size. The maximum growth rate, $\sim 0.39$ in this case, is slightly smaller than that for the vanishing radial wavelength, but the azimuthal wavelengths of the most unstable mode are similar in the two situations. Our simulations correspond to conditions somewhat between the dotted and dot-dashed lines.

It is interesting to note that while the pure interchange mode is stable, we see the characteristics of the interchange mode in the dispersion relation of the mixed mode. The reason is the following: When three-dimensional perturbations are applied to the initial system, it quickly adjusts itself by forming alternating regions of compression and rarefaction along the radial direction. Both alternating regions are now subject to the undular instability. For smaller azimuthal wavenumbers, the undular instability in each rarefied region develops faster with the help of buoyancy due to the neighboring dense regions. That is why the fastest growing mode has $k_{x}=\infty$.

\section{RESULTS}

\subsection{Effect of Resolution on Radial Wavenumber}

The dispersion relations in Figure 1 have shown that the most unstable mode has vanishing wavelengths along the radial direction. This has guided us to use the highest resolution (using up to $256^{3}$ grid zones) achievable with our computing resources. Even with the highest resolution, it is, however, not possible to resolve all the small-scale structures that are expected to form in the simulations. Bearing this fact in mind, we compare in Figure 2 isodensity surfaces at the same epoch $(t=35)$ but with different resolutions using $64^{3}, 128^{3}$, and $256^{3}$ zones (models 1,2 , and 3 , respectively). The computational boxes are oriented in such a way that the radial $(x)$, azimuthal $(y)$, and vertical $(z)$ directions are from left to right, from near to far, and from bottom to top, respectively. A gray surface with equal density $\rho_{0}(z=4)$ is included in each box, which was initially a flat surface at $z=4$. The first impression is that the density structure in the low-resolution $\left(64^{3}\right.$ zones) simulation looks very different from that in the high-resolution $\left(256^{3}\right.$ zones) simulation. Similarly, it looks like large-scale, ordered structures are formed in the low-resolution simulation. On the other hand, small-scale, chaotic structures are clear in the high-resolution simulation, which is expected from linear stability analyses. This justifies the usage of resources to perform high-resolution simulations. In what follows, we discuss only the results of the high-resolution simulations, models 3 and 4 .

Since the instability has been initiated by random perturbations, no preferred wavelength has been introduced, but the upper and lower limits of available wavelengths are set by the finite size of the computational box, which is $12 \mathrm{H}$, and the numerical resolution of the code, respectively. In general, the number of grid zones required to resolve one wavelength is different from problem to problem and from code to code. It is our experience that our code needs approximately eight grid zones along one direction to cover one wavelength in the current simulations without excessive numerical dissipation. With 256 zones, the smallest wavelength that can be resolved would be $\sim \frac{3}{8} H$, and $\sim 32$ wavy structures are accommodated along one direction.

\subsection{Time Evolution of rms Velocities}

Figure 3 plots the time evolution of the rms velocity (volume-averaged, not mass-averaged) in the simulations for the case without rotation $(\Omega=0$; left-hand panel) and with rotation $\left(\Omega=\frac{1}{2} ;\right.$ right-hand panel). The $x$-, $y$-, and $z$ components are drawn with dotted, short-dashed, and longdashed lines, respectively. The solid line corresponds to the maximum linear growth rate, 0.41 . During the initial transient stage $(0 \leq t \lesssim 25)$, random perturbations adjust themselves by generating one-dimensional magnetosonic waves,
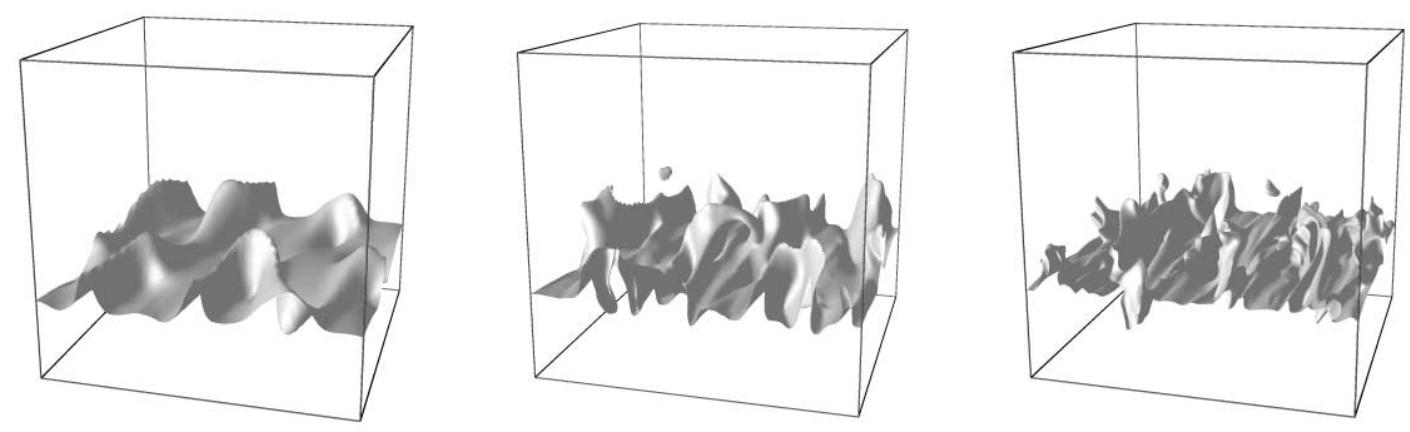

FIG. 2.-Comparison of the density structure simulated with different number of grid zones. Left: coarse (64 $4^{3}$, model 1); Middle: medium (128 ${ }^{3}$, model 2); and Right: high $\left(256^{3}\right.$, model 3). The computational box is oriented in such a way that the radial $(x)$, azimuthal $(y)$, and vertical $(z)$ directions are from left to right, from near to far, and from bottom to top, respectively. At the same epoch $t=35$, the gray surface with density $\rho_{0}(z=4)$ is shown. The unit of time is $H / a$, and the size of the computational box is $(12 H)^{3}$. 


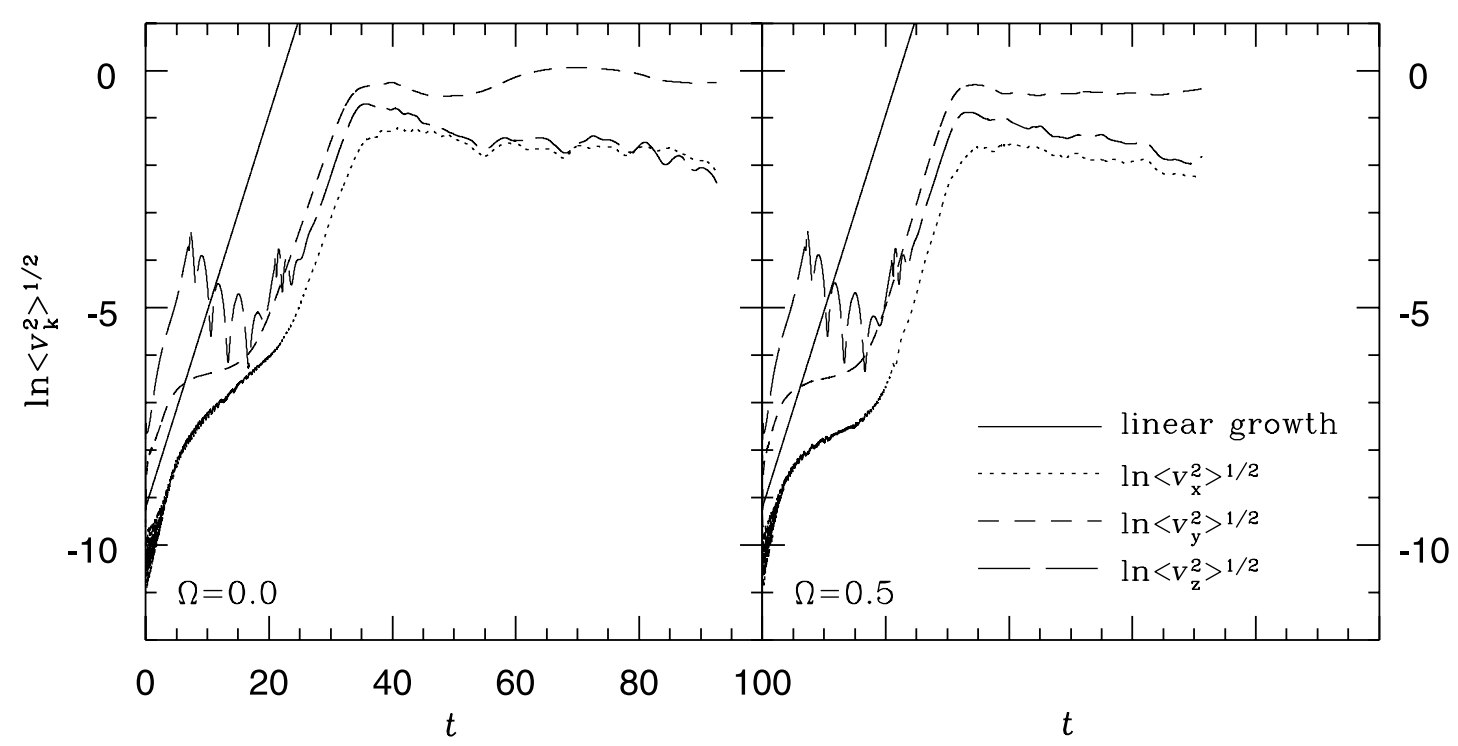

FIG. 3.-The rms of each velocity component as a function of time. The left-hand panel is for the case without rotation, and the right-hand panel is for the case with rotation. The natural log is used along the ordinate. The maximum linear growth rate, 0.41 , is represented by the solid line. The units of velocity and time are $a$ and $H / a$, respectively.

which move vertically, as pointed out in Kim et al. (1998) and Santillán et al. (2000). On top of the magnetosonic waves, there are slow MHD waves that eventually develop into the unstable mode (Shu 1974; Kim et al. 1997). As the slow MHD waves grow, the fluctuating and propagation features of the magnetosonic waves gradually diminish.

As in Kim et al. (1998), the evolution can be divided into three stages: linear, nonlinear, and relaxed. The linear stage lasts until $t \simeq 35$ for $\Omega=0$ and $t \simeq 32$ for $\Omega=\frac{1}{2}$. In both cases, the slope of the linear growth is close to 0.41 . This demonstrates that the numerical resolution is fine enough to follow the modes that have small radial wavelengths. At the end of the linear stage, the rms of $v_{y}$ is saturated near 0.7 , which is comparable to the isothermal sound speed. This implies that the linear approximation goes well beyond its conventional limit.

After saturation, the evolution enters the nonlinear stage. As pointed out in Kim et al. (1998), because of the manifestation of the interchange mode, the structures formed in the nonlinear stage are characterized as chaotic (see $\S 3.4$ ). On top of the chaotic structures, the undular mode causes magnetic field lines to drop at valley regions and rise at arch regions. Along those undulated field lines, gas supersonically falls toward valley bottoms and is stopped by shock waves. The gas ends up forming condensations at the valley bottoms. In the case without rotation, the condensations become overcompressed because of the continual falling of the gas. This is apparent in the left-hand panel of Figure 3 for $\Omega=0$ around $t \simeq 55$, when the rms of $v_{z}$ shows its local minimum. After that, the increased pressure pushes the valley matter back to the upper regions. This is recurrent but with the rms of $v_{z}$ decreasing. At the same time, reconnection, enhanced by chaotic motions, allows redistribution of mass and the magnetic field, and the system evolves into a new equilibrium state described in Kim et al. (1998) that is stable against the instability. For this reason, $t \gtrsim 55$ is labeled as the relaxed stage. However, in the case with rotation, the infalling gas speed is reduced by the Coriolis force, and so the signature of a local minima is not quite so dra- matic. Hence, distinguishing the relaxed stage from the nonlinear stage is less obvious.

A close examination of Figure 3 reveals the following further differences between the two cases: (1) The rms of $v_{y}$ reaches its peak value 0.78 at $t \simeq 40$ for $\Omega=0$, while the peak value reaches 0.74 at $t \simeq 35$ for $\Omega=\frac{1}{2}$. The difference is not large because the velocity has been small prior to the nonlinear stage, but it is the Coriolis force that makes the rms variations saturated at a lower level and at an earlier time epoch in the case with rotation. (2) The rms values of both $v_{x}$ and $v_{z}$ become converged in the case without rotation, while the rms of $v_{z}$ is always larger than that of $v_{x}$ in the case with rotation. This is because the Coriolis force affects only the horizontal flow.

\subsection{Energetics}

To examine the global properties of the instability further, we have followed the evolution of kinetic, magnetic, gravitational, and heat energies, which are defined as

$$
\begin{gathered}
E_{k}=\iiint \frac{1}{2} \rho\left(v_{x}^{2}+v_{y}^{2}+v_{z}^{2}\right) d x d y d z, \\
E_{m}=\iiint \frac{1}{8 \pi}\left(B_{x}^{2}+B_{y}^{2}+B_{z}^{2}\right) d x d y d z, \\
E_{g}=\iiint \rho \phi d x d y d z, \\
E_{h}=\iiint p \ln p d x d y d z,
\end{gathered}
$$

where the integrals cover the whole computation box, $\phi=g z$ is the gravitational potential, and $p=\rho a^{2}$ is the gas pressure (Mouschovias 1974). We take $\rho_{0}(0) a^{2} H^{3}$ as the normalization unit for the energies.

Time evolution of the normalized energies is plotted in Figure $4 a$ by solid lines for $\Omega=0$ and dotted lines for $\Omega=$ $\frac{1}{2}$. The general behavior has a distinct feature at each evolu- 


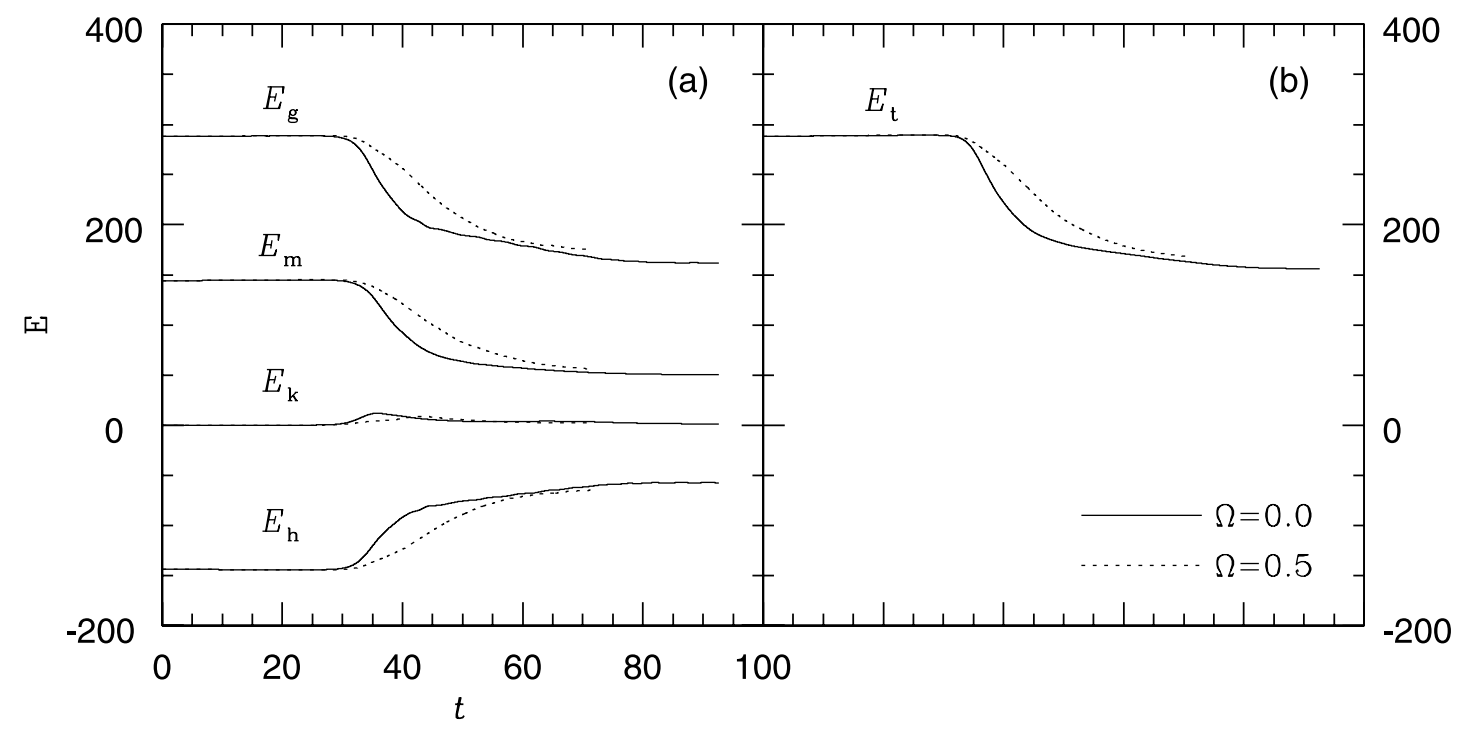

FIG. 4.-Evolution of $(a)$ kinetic $E_{k}$, magnetic $E_{m}$, gravitational $E_{g}$, and heat energies $E_{h}$ and $(b)$ the energy integral $E_{t}\left(=E_{k}+E_{m}+E_{g}+E_{h}\right)$. Energies for the case without rotation are represented by solid lines, whereas those with rotation are represented by dotted lines. The units of energy and time are $\rho_{0}(0) a^{2} H^{3}$ and $H / a$, respectively.

tionary stage. The energies remain unchanged during the linear stage, show significant variation during the nonlinear stage, and then slowly converge to final values in the relaxed stage. During the linear stage, velocity is less than the isothermal speed, and the Coriolis force is relatively small. So we cannot notice any visible difference in energies between the two cases with and without rotation. However, as the Coriolis force becomes stronger in the nonlinear stage, differences appear. The variation for $\Omega=\frac{1}{2}$ is less severe, showing a slower increase or decrease than that for $\Omega=0$. This is because the flow induced by the instability has been slowed down by the Coriolis force, but the increase or decrease of energies lasts longer. Hence, the energies eventually converge to the same final values in both cases in the relaxed stage. This implies that the final energetics of the instability are insensitive to rotation.

In Figure $4 a$, we see a bump in the curve of the kinetic energy $E_{k}$. It is barely noticeable, although the rms velocities are comparable to the isothermal speed during the nonlinear stage (see Fig. 3). This is because most flow activities either occur in the upper, low-density regions or are localized in the magnetic valleys. As gas in higher positions accumulates into the magnetic valleys, gravitational energy $E_{g}$ is released and becomes available to the system. Some of it is used to increase the kinetic energy and other converted to work done by gas. The heat energy $E_{h}$ measures the amount of this work (Mouschovias 1974). The total magnetic flux is conserved in our simulations because of the imposed boundaries, but the magnetic energy $E_{m}$ decreases. The reason is the following: As the instability develops, although it becomes compressed in the localized regions of magnetic valleys, the magnetic field, which is initially concentrated around the equatorial plane (see eq. [4]), spreads upward overall. This is because magnetic field lines manage to slip away mass because of enhanced reconnection. A given amount of magnetic flux that is less concentrated has less energy. The final, converged value of the magnetic energy will be the energy of the uniformly distributed magnetic field whose flux is same as the initial flux.
Mouschovias (1974) defined the energy integral of the system described in $\S 2.1$ as

$$
E_{t}=E_{k}+E_{m}+E_{g}+E_{h}
$$

and showed that it is a constant of motion. That is, the energy released from certain forms should end up increasing the energy in other forms. Matsumoto et al. (1990) showed, however, that the energy integral in their simulations of the Parker instability in a point mass-dominated gravity is not a conserved quantity when shocks are generated. Our simulations have confirmed this. The curves labeled with $E_{t}$ in Figure $4 b$ are constant up to the end of the linear stage since no shock has been developed yet. However, as soon as the nonlinear stage begins (so the increase in the kinetic energy becomes noticeable), shocks form in the valley regions, so the energy integral starts to decrease. When the system enters the relaxed stage, it gradually settles to the final equilibrium state in which all shocks have disappeared. Then the energy integral stays constant again.

\subsection{Structures}

To illustrate the structures resulting from the instability, three-dimensional images are shown in Figure 5. The perspective images of density structure and magnetic field lines are at two epochs of the linear stage ( $t=30$; upper panels) and the nonlinear stage ( $t=36$; lower panels), respectively. Only the images for $\Omega=\frac{1}{2}$ are presented since the corresponding images for $\Omega=0$ look similar. Also refer to the images for $\Omega=0$ in Figure 2 and in Kim et al. (1998), although they are drawn at different epochs. A gray surface with density $\rho_{0}(z=4)$ is depicted in each left-hand panel, as in Figure 2, while 64 field lines, whose starting points lie along the line of $z=4$ and $y=0$, are drawn in each righthand panel. At $t=0$, the field lines are straight and lie in the $z=4$ plane.

As mentioned in $\S 2.2$, in the linear stage, the interchange mode induces alternating regions of compression and rarefaction along the $x$-direction, while the undular mode bends magnetic field lines vertically. Those features are 

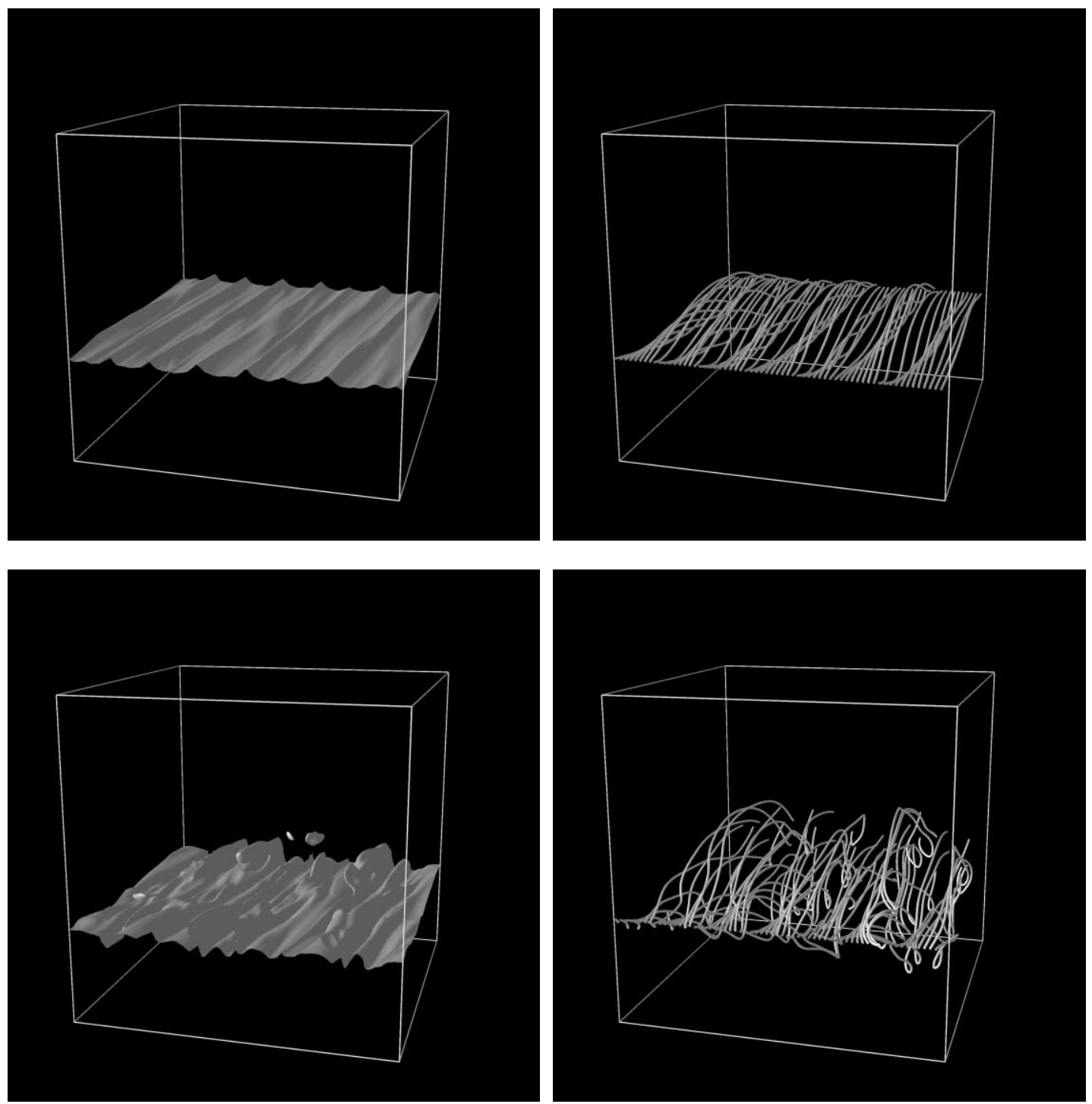

Fig. 5.-Perspective view of the density structure and magnetic field lines at the epochs $t=30$ (top panel) and $t=36$ (bottom panel) from the highresolution simulation with rotation (model 4). The computational boxes are oriented in the same way as in Fig. 2 . The gray surface with isodensity $\rho_{0}(z=4)$ is shown in the left-hand panels. Magnetic field lines (64), whose starting points lie along the line of $z=4$ and $y=0$, are represented by the green tubes in the right-hand panels. The units of length and time are $H$ and $H / a$, respectively. [See the electronic edition of the Journal for a color version of this figure.]

clearly seen in the images of the linear stage. The isodensity surface in the upper left-hand panel of Figure 5 is corrugated along the $x$-direction, which is the defining feature of the interchange mode. The field lines in the upper righthand panel go up and down along the $y$-direction, making valley and arch regions, which are the distinguishing features of the undular mode. As the instability develops, the alternating structures become complicated. At the same time, the density contrast between the valley and arch regions becomes large. The isodensity surface in the lower left-hand panel shows bumps at the valley and dents at the arch regions. There is no regular occurrence pattern of the bumps and dents even though the corrugations of the surface along the $x$-direction are still preserved in this stage. The distribution of the field lines becomes complicated too. As more gas moves along field lines from the arch to the valley, the arch field lines move to even higher positions and the valley field lines move down to lower positions, as clearly shown in the lower right-hand panel.
An effect of rotation on nonlinear structures is illustrated in Figure 6, where two field lines together with velocity vectors along them are plotted at $t=40$. Both are examples of valley and arch field lines. Flow velocity along those undulated field lines becomes large in the nonlinear stage. So flows are under the strong influence of the Coriolis force. As gas infalls along the valley field line, flow velocity increases and reaches its maximum around the bottom of the valley. The near-side gas along the left field line in Figure 6 experiences the force in the positive $x$-direction, while the far-side gas experiences the oppositely directed force. These forces induce the largest oppositely twisting velocities around the bottom, which cause the bottom part of the undulated field line to become twisted. A similar situation occurs along the arch field line in the right-hand side of Figure 6. This time, the near-side gas from the arch is deflected toward the negative $x$-direction, and the far-side gas is deflected toward the positive $x$-direction. However, the flow velocity is smaller around the tip of the arch, so the 


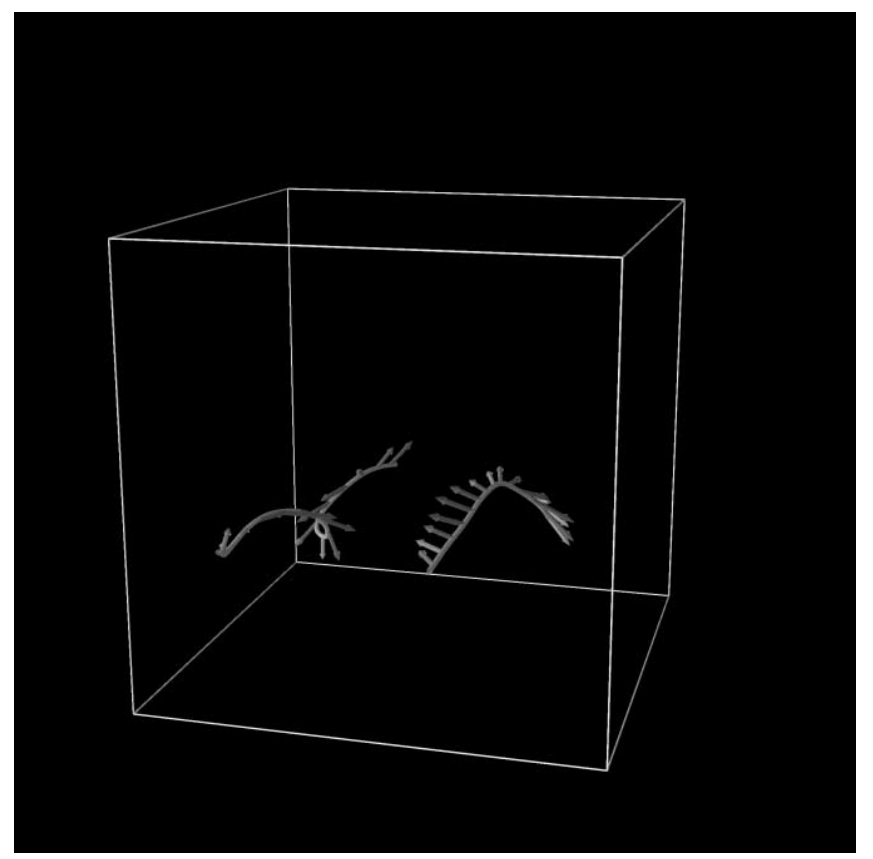

FIG. 6.-Two magnetic field lines and the velocity vectors along them at the epoch $t=40$ from the high-resolution simulation with rotation (model 4). The computational box is oriented in the same way as in Fig. 2. The field lines are drawn in green, and the velocity vectors are drawn in red. The normalization units are the same as in Fig. 5. [See the electronic edition of the Journal for a color version of this figure.]

field line is not twisted. Comparing the field lines at the valley and arch regions, we find that the curvature of the arch field is larger than that of the valley field.

Note that we have started our simulations only with the uniform magnetic field, but the field lines, which have been bent in magnetic valley regions, get twisted because of the Coriolis force. Since the direction of the twisted lines changes rapidly, they may be regarded as a "random" field. So the Coriolis force has played a role of converting the uniform component of magnetic field into the random one. Assuming that the scale of the twisting is comparable to the size of condensations formed in valley regions, $100 \mathrm{pc}$ (see the next subsection), it is not far from the single-cell size of the Galactic random field measured in Rand \& Kulkarni (1989).

\subsection{Column Density}

The structures formed are further illustrated by examining the column densities defined by

$$
\begin{aligned}
& N_{z}(x, y ; t)=\frac{\int_{0}^{12} \rho(x, y, z ; t) d z}{\int_{0}^{12} \rho(x, y, z ; 0) d z}, \\
& N_{x}(y, z ; t)=\frac{\int_{0}^{12} \rho(x, y, z ; t) d x}{\int_{0}^{12} \rho(x, y, 0 ; 0) d x}, \\
& N_{y}(x, z ; t)=\frac{\int_{0}^{12} \rho(x, y, z ; t) d y}{\int_{0}^{12} \rho(x, y, 0 ; 0) d y} .
\end{aligned}
$$

We have kept track of the evolution of the vertical column density $N_{z}$ and plotted in Figure 7 its minimum and maximum as functions of time. The solid lines correspond to $\Omega=0$ and the dotted lines to $\Omega=\frac{1}{2}$. Both cases show a similar trend of evolution. Initially, $N_{z}$ stays close to unity for all values of $x$ and $y$. As gas starts to fall into valleys in the linear stage, the contrast between the minimum and

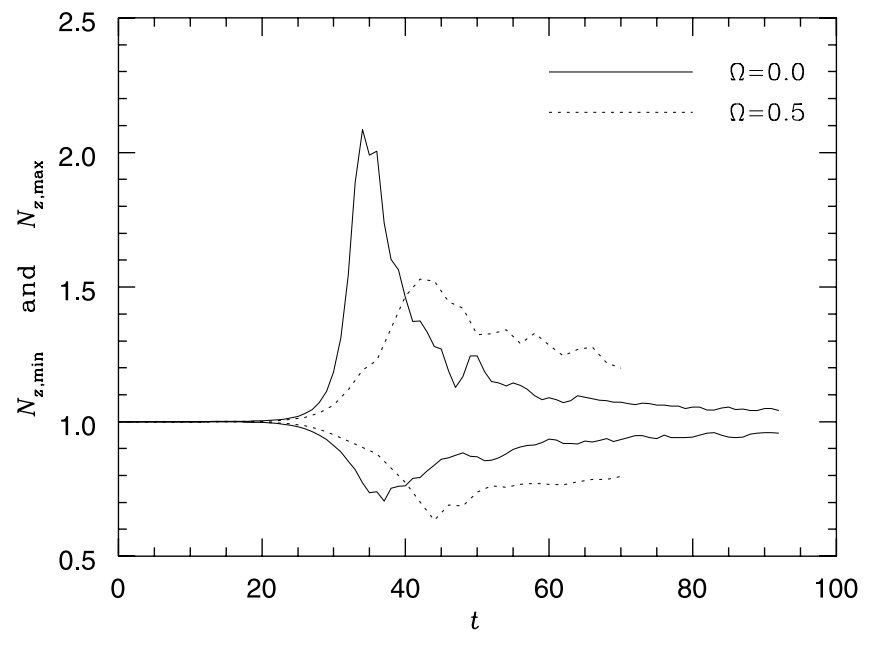

FIG. 7.-Maximum and minimum of the vertical column densities as functions of time. The column density has been normalized to its initial value. The solid lines represent the case without rotation and the dotted ones the case with rotation. The unit of time is $H / a$.

maximum increases. It reaches peaks in the nonlinear stage and decreases during later evolution. In addition, there are differences between the two cases: (1) the peak of the maximum $N_{z}$ for $\Omega=\frac{1}{2}$ (1.5) is smaller than that (2.1) for $\Omega=0$, indicating that the nonlinear structures are less acute, (2) the maximum $N_{z}$ for $\Omega=\frac{1}{2}$ peaks at later time, indicating that the nonlinear structures develop more slowly, and (3) the maximum $N_{z}$ for $\Omega=\frac{1}{2}$ is broader, indicating that the nonlinear activity lasts longer. All these are attributed to the effects of the Coriolis force and agree with the descriptions in $\S \S 3.2$ and 3.3.

At $t=35$ for $\Omega=0$ and $t=42$ for $\Omega=\frac{1}{2}$ around the peaks of the maximum $N_{z}$, the distributions of $N_{z}$ in the $x-y$-plane are drawn in Figure 8. In each panel, equal column density contours overlie a gray-scale map. The thick solid lines that correspond to $N_{z}=1$ trace out the boundaries of over- and underdense regions. Overdense regions are shown as thin solid lines and dark gray regions, whereas underdense regions are shown as dotted lines and light gray regions. In the case without rotation (see Fig. 8a), sheetlike structures form whose long dimension is aligned with the initial field $(y)$ direction, as pointed out in Kim et al. (1998). Similar structures are formed in the case with rotation, but the long dimension is a bit tilted with respect to the $y$-direction, and the shape is a bit rounder (see Fig. $8 b$ ). The tilting is, of course, the consequence of the Coriolis force, $-2 \Omega \hat{z} \times v$. Note that because of the periodic nature of our boundaries, the allowed angle for tilting is quantized and given by

$$
\theta_{t} \equiv \arctan \left(k_{y} / k_{x}\right),
$$

where the $k$ 's can have the values of $2 \pi / 12 H, 2(2 \pi / 12 H) \ldots$, $256(2 \pi / 12 H)\left(12 H\right.$ is the box size). Figure $8 b$ shows that $\theta_{t}$ is close to $\arctan \frac{1}{6}$, corresponding to $k_{x}=6(2 \pi / 12 H)$ and $k_{y}=2 \pi / 12 H$. Those coincide with $\left(k_{x}, k_{y}\right)$ of the structure most visible in the figure.

While the $N_{z}$ map illustrates the vertically developed structures, $N_{x}$ and $N_{y}$ are more closely compared with observations in the Galaxy. Considering that the initial field direction is parallel to $y$-direction and that the sheetlike structure is almost aligned with that direction, $N_{x}$ and $N_{y}$ represent two extremes: the line of sight is perpendicular 

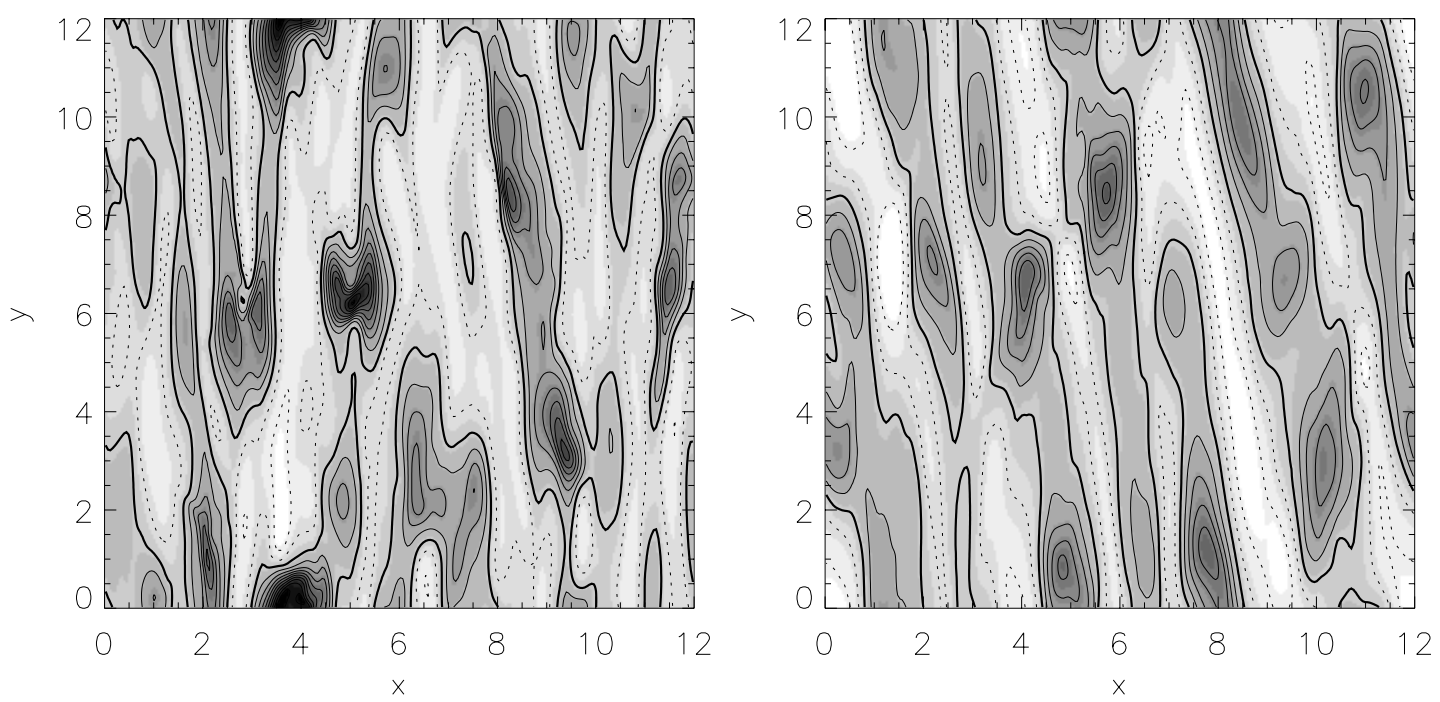

FIG. 8.-Vertical column density maps for the cases without rotation at $t=35$ (left) and with rotation at $t=42$ (right). The time epochs have been chosen so that the column density reaches peaks at those epochs. The gray-scale image of the column density is overlaid with equal density contours. The solid and dotted lines represent overdense and low-dense regions, respectively, which are divided by thick solid lines. The interval is $1 / 10$ of the initial value. The units of length and time are $H$ and $H / a$, respectively.

$\left(N_{x}\right)$ to and parallel $\left(N_{y}\right)$ to the mean field direction. The observed column density would be somewhere between the two. Contours with equal $N_{x}$ and $N_{y}$ are plotted in the leftand right-hand panels of Figure 9, respectively, for the case with rotation at $t=36$. We have chosen 10 contours whose column densities are $e^{-1}, e^{-2}, \ldots, e^{-10}$ of the initial midplane value. So initially the contours were straight and equally spaced. Each contour in the left-hand panel shows one or two bumps along the $y$-direction, which are attributed to the manifestation of the undular mode. On the other hand, the counterpart in the right-hand panel is more irregular and shows many bumps along the $x$-direction, which are attributed to the manifestation of the interchange mode.

\section{SUMMARY AND DISCUSSION}

We have studied the evolution of the Parker instability in nonrotating and uniformly rotating magnetized disks

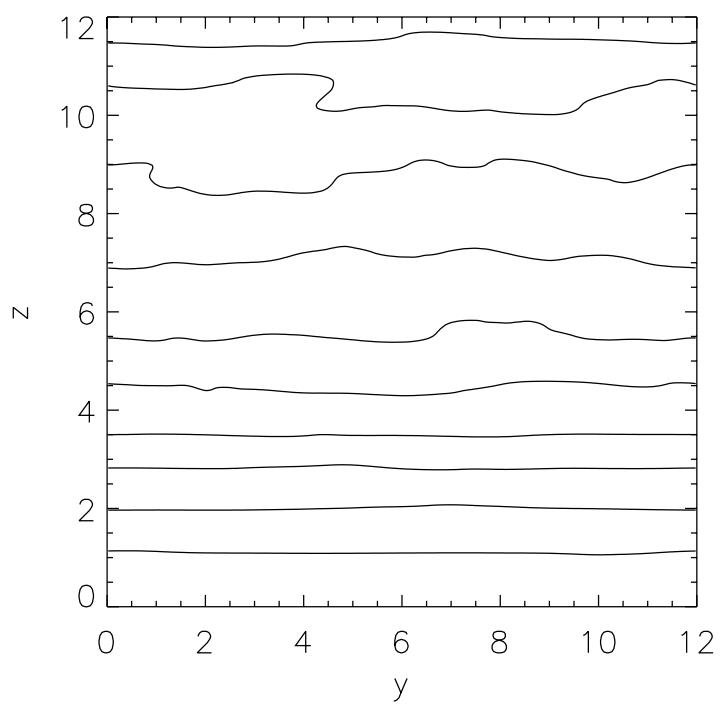

through high-resolution numerical simulations (using up to $256^{3}$ grid zones). The main purpose of this paper is to address the effects of rotation on the instability by comparing the two cases. Here our findings are summarized.

First, the overall evolution of the instability in a uniformly rotating disk is similar to that in a nonrotating disk. As in the case without rotation (Kim et al. 1998), the evolution in the case with rotation is divided into three stages: linear, nonlinear, and relaxed. In the linear stage, the mixed mode regulates the evolution, as predicted in linear stability analyses. That is, exponentially growing perturbations have a preferred scale along the azimuthal direction but have the smallest possible scale along the radial direction. In the nonlinear stage, the growth is saturated. The undular mode bends magnetic field lines, forming valleys and arches, but overall the flow motions appear chaotic. After becoming fully developed, the nonlinear oscillatory structures damp out, and at the same time, reconnection enhanced by

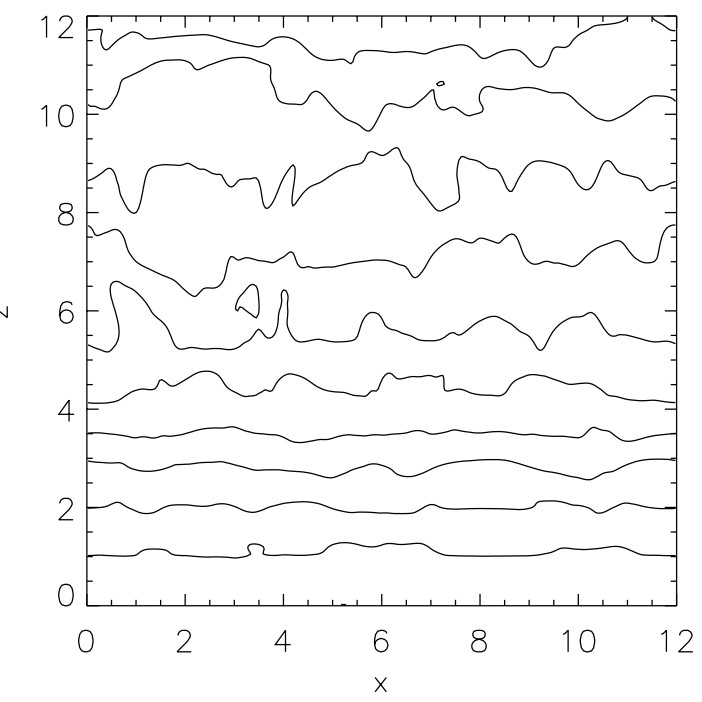

FIG. 9.- Horizontal column density maps for the case with rotation at $t=36$. The density has been integrated along the radial direction in the left-hand panel and along the azimuthal (mean magnetic field) direction in the right-hand panel. Ten equal column density contours represent the lines with $e^{-1}, e^{-2}$, $\ldots, e^{-10}$ of the initial midplane value. The units of length and time are $H$ and $H / a$, respectively. 
chaotic motions allows redistribution of the mass and the magnetic field. At this stage, the system enters the relaxed stage, although the boundary between the nonlinear and relaxed stages is not as clear as in the case without rotation.

Second, the Coriolis force introduces a couple of visible differences. One of them is the twisting of magnetic field lines in valley regions. In the case with rotation, gas is subject to the Coriolis force whenever there are lateral motions, but the force is especially strong in valley regions because the falling speed of gas toward those regions becomes comparable to or faster than the isothermal sound speed. Flows approaching valleys from two sides along the field lines experience the oppositely directed Coriolis forces, so the field lines there get twisted. The scale of the twisting is comparable to the size of the valleys and is $\sim 100 \mathrm{pc}$ or less. It is noticeable that this scale of twisting is compatible with the "single-cell size" of random magnetic field measured by Rand \& Kulkarni (1989).

Third, we have found that the maximum enhancement factors of vertical column density are 2.1 for the case without rotation and 1.5 for the case with rotation. In the former case, the enhanced sheetlike structures, viewed from the vertical column density map, are aligned with the direction of the mean magnetic field. On the other hand, in the latter case, the structures are slightly tilted with respect to the field direction and a bit rounder. This is because the lateral motions of gas are affected by the Coriolis force.

In our previous study of Kim et al. (1998), we concluded that it is difficult to regard the Parker instability alone as the formation mechanism of GMCs because (1) the sheetlike shape of the structures formed is different from the shape of GMCs and (2) the density enhancement factor is too small. The inclusion of rotation does not change this conclusion. However, we do not completely rule out the role of the Parker instability in the formation of GMCs. The inclusion of radiative cooling and self-gravity may result in thermal and gravitational instabilities, which certainly increase the density enhancement factor far more than the one brought by the Parker instability alone and shape structures in a different way. In the context of a more complicated system with cooling and self-gravity, the Parker instability agglomerates diffuse gas into magnetic valley regions, which provide a more conducive environment to both thermal and gravitational instabilities. Afterward, they may override the Parker instability, especially in each magnetic valley region, and govern later evolution. Here we want to emphasize that other physical processes, such as thermal and gravitational instabilities, are required to get more than factor of 2 increase in vertical column density.

Because of the sheetlike shape of the nonlinear structures aligned with the mean magnetic field, the horizontal column density, which is an observable quantity in the Galaxy, depends on the angle between the line of sight and the field direction. When they are aligned with each other, the column density map illustrated in the right-hand panel of Figure 9 shows vertical filamentary structures. So we suggest that the Parker instability would be a possible mechanism for the formation of the interstellar filamentary structures. The "filament 1 " revealed in an $\mathrm{H} \alpha$ survey (Haffner, Reynolds, \& Tufte 1998) is the most plausible observed candidate that may have been induced by the Parker instability. It is about $1 \mathrm{kpc}$ long, has an arc-shaped morphology, and resides at high latitude. These are essentially the attributes of the Parker instability. It is, however, difficult to make a direct comparison between our simulations and their observations because the Galactic environment at the location (about $1 \mathrm{kpc}$ away from the Sun to the direction of $l=225^{\circ}$ ) of filament 1 could be different from that in the solar neighborhood. It may be an interesting project to perform a direct numerical modeling for filament 1 .

In this paper, we have studied the effects of uniform rotation, although differential rotation is a proper description of the Galactic rotation law. Inclusion of differential rotation into magnetized gas disks would complicate the situation because not only the Parker instability but also the magnetorotational instability (MRI; Balbus \& Hawley 1991; Hawley \& Balbus 1991) can come into existence. The evolution of the two instabilities in accretion disks was studied, for instance, by Miller \& Stone (2000) through threedimensional simulations. However, the MRI in the Galactic disk is not as active as in accretion disks and can even be stabilized because the Galactic magnetic field is very strong, but it is not clear whether the MRI is completely suppressed for the following reason: As the Parker instability proceeds, high- $\alpha$ arch regions and low- $\alpha$ valley regions are segregated. The arch regions are stable to the MRI because of even stronger magnetic fields, but the valley regions may be subject to the MRI. The action of the two instabilities on the magnetic field is different. The Parker instability pumps field lines vertically, while the MRI stretches them radially. Together these instabilities and differential rotation along with dissipation and/or reconnection mechanisms of the magnetic field may serve as the ingredients of the Galactic dynamo (Tout \& Pringle 1992). This line of approach to the Galactic dynamo would be worthy of further detailed investigations.

We would like to thank Don Cox and Bob Benjamin, who reviewed this paper. They picked up a mistake in Figure 4 and provided several suggestions/comments that improved the paper. The work by D. R. was supported in part by the grant KRF 99-015-DI0113. The work by T. W. J. was supported in part by the NSF through grants INT 95-11654 and AST 96-19438, by the NASA grant NAG 5-5055, and by the University of Minnesota Supercomputing Institute.

\section{REFERENCES}

Appenzeller, I. 1974, A\&A, 36, 99

Baierlein, R. 1983, MNRAS, 205, 669

Balbus, S. A., \& Hawley, J. F. 1991, ApJ, 376, 214

Basu, S., Mouschovias, T. Ch., \& Paleologou, E. V. 1997, ApJ, 480, L55

Blitz, L., \& Shu, F. H. 1980, ApJ, 238, 148

Chou, W., Tajima, T., Matsumoto, R., \& Shibata, K. 1997, PASJ, 49, 389

Falgarone, E., \& Lequeux, J. 1973, A\&A, 25, 253

Feast, M., \& Whitelock, P. 1997, MNRAS, 291, 683

Foglizzo, T., \& Tagger, M. 1994, A\&A, 287, 297

-. 1995, A\&A, 301, 293
Giz, A. T., \& Shu, F. H. 1993, ApJ, 404, 185

Gomez de Castro, A. I., \& Pudritz, R. E. 1992, ApJ, 395, 501

Haffner, L. M., Reynolds, R. J., \& Tufte, S. L. 1998, ApJ, 501, L83

Hanawa, T., Matsumoto, R., \& Shibata, K. 1992, ApJ, 393, L71

Handa, T., Sofue, Y., Ikeuchi, S., Kawabe, R., \& Ishizuki, S. 1992, PASJ, 44, L227

Hawley, J. F., \& Balbus, S. A. 1991, ApJ, 376, 223

Heiles, C. 1996, in ASP Conf. Ser. 97, Polarimetry of the Interstellar Medium, ed. W. G. Roberge \& D. C. B. Whittet (San Francisco: ASP), 457 
Horiuchi, T., Matsumoto, R., Hanawa, T., \& Shibata, K. 1988, PASJ, 40, 147

Hughes, D. W., \& Cattaneo, F. 1987, Geophys. Astrophys. Fluid Dyn., 39,

Kaisig, M., Tajima, T., Shibata, K., Nozawa, S., \& Matsumoto, R. 1990, ApJ, 358, 698

Kamaya, H., Horiuchi, T., Matsumoto, R., Hanawa, T., Shibata, K., \& Mineshige, S. 1997, ApJ, 486, 307

Kim, J., Franco, J., Hong, S. S., Santillán, A., \& Martos, M. A. 2000, ApJ, 531,873

Kim, J., \& Hong, S. S. 1998, ApJ, 507, 254

Kim, J., Hong, S. S., \& Ryu, D. 1997, ApJ, 485, 228

Kim, J., Hong, S. S., Ryu, D., \& Jones, T. W. 1998, ApJ, 506, L139

Kim, J., Ryu, D., Jones, T. W., \& Hong, S. S. 1999, ApJ, 514, 506

Matsumoto, R., Horiuchi, T., Hanawa, T., \& Shibata, K. 1990, ApJ, 356, 259

Matsumoto, R., \& Shibata, K. 1992, PASJ, 44, 167

Matsumoto, R., Tajima, T., Shibata, K., \& Kaisig, M. 1993, ApJ, 414, 357

Miller, K. A., \& Stone, J. M. 2000, ApJ, 534, 398
Mouschovias, T. Ch. 1974, ApJ, 192, 37

Mouschovias, T. Ch., Shu, F. H., \& Woodward, P. R. 1974, A\&A, 33, 73

Nozawa, S., Shibata, K., Matsumoto, R., Sterling, A. C., Tajima, T., Uchida, Y., Ferrari, A., \& Rosner, R. 1992, ApJS, 78, 267

Parker, E. N. 1966, ApJ, 145, 811

. 1967, ApJ, 149, 535

Rand, R. J., \& Kulkarni, S. R. 1989, ApJ, 343, 760

Ryu, D., \& Jones, T. W. 1995, ApJ, 442, 228

Ryu, D., Jones, T. W., \& Frank, A. 1995, ApJ, 452, 785

Santillán, A., Kim, J., Franco, J., Martos, M., Hong, S. S., \& Ryu, D. 2000, ApJ, 545, 353

Shibata, K., \& Matsumoto, R. 1991, Nature, 353, 633

Shibata, K., Tajima, T., Matsumoto, R., Horiuchi, T., Hanawa, T., Rosner, R., \& Uchida, Y. 1989a, ApJ, 338, 471

Shibata, K., Tajima, T., Steinolfson, R. S., \& Matsumoto, R. 1989b, ApJ, 345,584

Shu, F. H. 1974, A\&A, 33, 55

Tout, C. A., \& Pringle, J. E. 1992, MNRAS, 259, 604

Zweibel, E. G., \& Kulsrud, R. M. 1975, ApJ, 201, 63 